\title{
Visible near Infrared Spectroscopic Evidence of the Purification and Dispersion of Single-Walled Carbon Nanotubes
}

Jinling Gao*, Mingjie Shen, Dezhao Hua, Xiaoxue Gao

\author{
College of Science, Heilongjiang Bayi Agricultural University, Daqing, 163319, China
}

DOI: $\underline{10.36347 / \text { sipms.2020.v07i08.003 }}$

| Received: 09.08.2020 | Accepted: 17.08.2020 | Published: 19.08.2020

*Corresponding author: Jinling Gao

Abstract

Original Research Article

The application of dispersible single-walled carbon nanotubes in water is required in many fields, such as mechanics, thermology, electricity, etc. In this paper, single-walled carbon nanotube samples were dispersed in sodium deoxycholate aqueous solution with the assistance of ultrasonic crushing. The effects of centrifugal time, concentration and centrifugal velocity on dispersion were investigated. After centrifugal separation, the dispersible single-walled carbon nanotubes solution in water was obtained. The dispersion behavior of single-walled carbon nanotubes in sodium deoxycholate aqueous solution was further investigated by Uv-visible near infrared spectrum and electrochemical cyclic voltammetry curve. At the same time, characterization results of Uv-visible near infrared spectrum shew that the purity of single-walled carbon nanotubes was improved. The cyclic voltammetry curves of single-walled carbon nanotubes after purification and dispersion were tested by electrochemical method, and the electron transfer behavior of single-walled carbon nanotubes after purification and dispersion was investigated.

Keywords: Single-walled carbon nanotubes; Uv-visible near infrared spectrum; Dispersion; Sodium deoxycholate. Copyright @ 2020: This is an open-access article distributed under the terms of the Creative Commons Attribution license which permits unrestricted use, distribution, and reproduction in any medium for non-commercial use (NonCommercial, or CC-BY-NC) provided the original author and source are credited.

\section{INTRODUCTION}

Single-wall carbon nanotubes (SWCNTs) have unique electrical properties, mechanical properties and optical properties [1]. But due to the van der Waals forces between the SWCNTs bundles, SWCNTs are commonly bunched, and their insolubility and difficult dispersion in aqueous media, which further severely limited their wide application in many fields. Therefore, the preparation of highly dispersible SWCNTs in water is a meaningful work.

In recent years, many researches have used various organic and inorganic solvents, surfactant molecules and biological molecules as dispersants to solve this problem. Materials, such as surfactants, ionic liquids, biomolecules, and polymers commonly were used to disperse SWCNTs. Syrgiannis, Z. found that polyoxometalates(POM) had different dispersing capacities for small diameter SWCNTs, this result chould be derived from the interaction POM and SWCNTs [2]. Ranran Wang reported that SWCNTs were dispersed by pristine DNA, which had better dispersion performance for SWCNTs, and the DNA/SWCNTs were prepared transparent conductive films on PET substrates, the films with high performance and good stability had been obtained [3].
Matsumoto, K. used ketone solvents to disperse SWCNTs, a stable dispersion system was obtained [4]. A kind of polymer ionic liquids PIL, which synthesized by three steps, was used to disperse SWCNTs, SWCNTs can be dispersed by PIL in DMF, and a sensing materials was made with ionic liquid package SWCNTs, a super sensitive $\mathrm{CO}_{2}$ sensor is developed, its detection limit is 500 PPT, and the sensor of $\mathrm{CO}_{2}$ has good selectivity [5]. The dispersion of SWCNTs by biological macromolecules was reported, and the structure and stability of the chiral index $(6,5)$ ssDNASWCNTs complex was studied by using the all-atom molecular dynamics simulation method. The dispersion efficiency of SWCNTs by ssDNA, flavonoids mononucleotide surfactants, and polyaminoamine (PAMAM) dendrimers was also compared [6].

In this paper, sodium deoxycholate was used as dispersing agent, single-walled carbon nanotube samples were dispersed in sodium deoxycholate aqueous solution with the assistance of ultrasonic, the dispersion behavior of single-walled carbon nanotubes in sodium deoxycholate aqueous solution was further investigated by Uv-visible near infrared spectrum, the electron transfer behavior of single-walled carbon nanotubes after purification and dispersion was 
investigated by electrochemical cyclic voltammetry curve.

\section{MATERIALS AND METHODS Materials and reagents}

Ac-discharged SWCNTs which were made and purified in laboratory, Ethanol, sodium deoxycholate and potassium hexyanide ferrate were analytical pure.

\section{Instruments and equipment}

Supercentrifuge (CP100WX, HITACHI of Japan), vacuum drying oven(DGF-6030, the company of Shanghai yiheng scientific instrument), electronic analytical balance (ALC-310.2, LTD. co. Beijing taike instrument), circulating water multi-purpose vacuum pump(SHB-IIIA, LTD. co. zhengzhou Great Wall science and trade), constant temperature magnetic stirring water bath(HJ-A2, LTD. co. changzhou maikonuo instrument), Uv-visible spectrophotometer(Uv-3600, HITACHI, Japan), electrochemical workstation(SP-300, Japan electronics).

\section{METHODS}

\section{The purification and Dispersion of SWCNTs}

SWCNTs were dispersed by sodium deoxycholate in accordance with the method described in literature [7]. Sodium deoxycholate $(1 \mathrm{mg})$ was added in a beaker $(150 \mathrm{~mL})$, deionized water $(100 \mathrm{~mL})$ was put to dissolve sodium deoxycholate, the solution was dispersed with ultrasonic until the solution was transparent. Then SWCNTs samples (5 mg) were added in the solution of sodium deoxycholate, it was dispersed for $12 \mathrm{~h}$ at $5^{\circ} \mathrm{C}$, the solution was put in some centrifuge tubes and put in a high-speed centrifuge, at the conditions of rotating speed was at $20000 \mathrm{rpm}$, temperature was at $10{ }^{\circ} \mathrm{C}$, the centrifugal separation was for $1 \mathrm{~h}$. At last, the top $80 \%$ solution was carefully removed with a syringe, the dispersed solution of
SWCNTs was obtained, the purification of SWCNTs was realized in the meanwhile.

\section{Construction of three-electrode working system}

According to the method in literature [8], PBS

buffer solution $(100 \mathrm{~mL}, \mathrm{pH}=7.4)$ was prepared, a certain amount of potassium hexanocyanate was added to the buffer solution, a buffer solution (Fe(III)/Fe(II)) was obtained. The glassy carbon electrode was polished by $\mathrm{Al}_{2} \mathrm{O}_{3}$, the glassy carbon electrode was washed with alcohol and deionized water in turn. Then the glassy carbon electrode was blow-dried with high purity nitrogen. The purified and dispersed SWCNTs was carefully aspirated onto the glassy carbon working electrode with a micro syringe. The modified working electrode was obtained and placed to dry. Then the auxiliary electrode (platinum wire electrode), reference electrode (saturated calomel electrode) and working electrode modified with purified and dispersed SWCNTs were put into the prepared PBS buffer solution, three-electrode working system was obtained. Cyclic voltammetry characteristics of working electrode modified with sodium deoxycholate-SWCNTs were investigated by electrochemical workstation testing, and electron transport of purified and dispersed SWCNTs was investigated.

\section{RESULTS AND ANALYSIS}

The purification and dispersion of SWNTs

The effect of sodium deoxycholate concentration on the dispersion of SWNTs

Ultraviolet near infrared spectroscopy is an important method to investigate the purification and dispersion of SWCNTs, so the purification and dispersion effect of SWCNTs were investigated by the absorption peak intensity of Ultraviolet near infrared spectroscopy. Figure-1 is the Vis-NIR spectra of dispersion solution of SWNTs with different concentration sodium deoxycholate(a) $0.5 \%$, (b) $1.0 \%$ and (c) $1.5 \%$

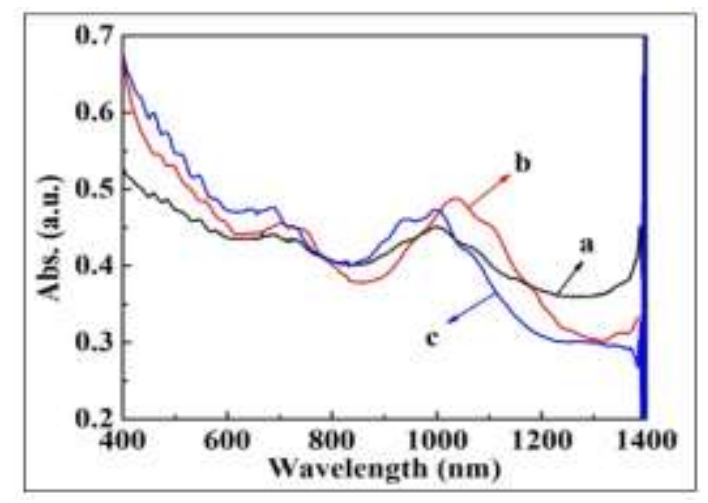

Fig-1: Vis-NIR spectra of dispersion solution of SWNTs with different concentration sodium deoxycholate(a) $0.5 \%$, (b) $1.0 \%$ and (c) $1.5 \%$

As is shown in figure 1, the Vis-NIR spectra of the solution of sodium deoxycholate-SWNTs varies with the concentration of sodium deoxycholate. The absorption spectra line (b) presents better resolution and higher effective absorption intensity than those of the absorption spectra line (a) and (c), the relative absorption peak of line (b) is larger than those of line (a) and (c). So the solution of sodium deoxycholate $(1 \%)$ has a better dispersion effect on SWNTs. It means the dispersion of sodium deoxycholate towards SWNTs 
is related to the concentration of sodium deoxycholate, when the concentration of sodium deoxycholate is $1 \%$, it has the best dispersion ability to SWNTs. When the concentration of sodium deoxycholate is $1 \%$, the purification of SWCNTs was higher, the purification of SWCNTs was realized in the meanwhile.
The effect of centrifugal speed on the dispersion of SWNTs

Figure-2 is the Vis-NIR spectra of dispersion solution of SWNTs with different centrifugal speed (a) $15000 \mathrm{rpm}$, (b) $20000 \mathrm{rpm}$ and (c) $25000 \mathrm{rpm}$.

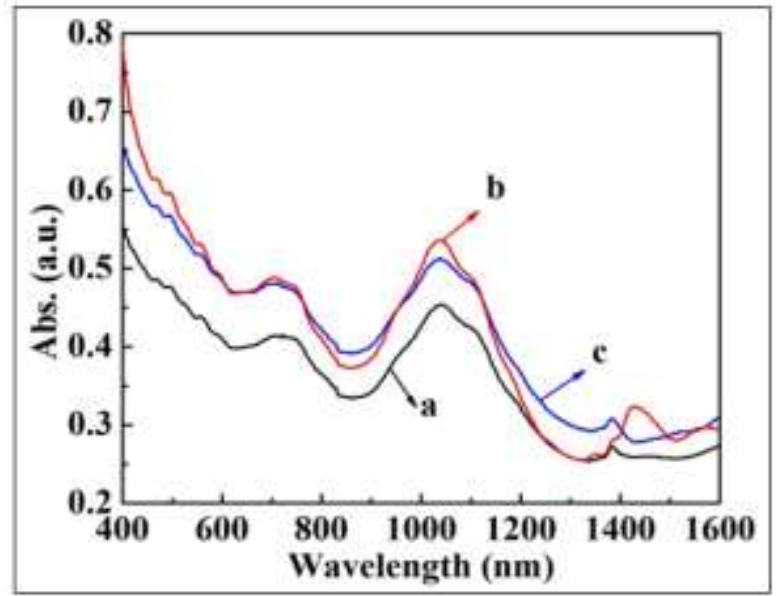

Fig-2: Vis-NIR spectra of dispersion solution of SWNTs with different centrifugal speed (a) $15000 \mathrm{rpm}$, (b) $20000 \mathrm{rpm}$ and (c) $25000 \mathrm{rpm}$

As is shown in Figure-2, the absorbance of the solution of sodium deoxycholate-SWNTs varies with the centrifugal speed. The absorption spectra line (b) presents better resolution and higher effective absorption intensity than those of the absorption spectra line (a) and (c), the relative absorption peak of line (b) is larger than those of line (a) and (c). So the centrifugal speed $(20000 \mathrm{rpm})$ has a better dispersion effect on SWNTs. It means the dispersion of sodium deoxycholate towards SWNTs is related to the centrifugal speed, when the centrifugal speed is 20000 $\mathrm{rpm}$, it has the best dispersion to SWNTs. When the centrifugal speed is $20000 \mathrm{rpm}$, the purification of SWCNTs was higher, the purification of SWCNTs was realized in the meanwhile.

\section{The effect of centrifugal time on the dispersion of} SWNTs

Figure-3 is the Vis-NIR spectra of dispersion solution of SWNTs with different centrifugal time (a) 1 h, (b) $1.5 \mathrm{~h}$ and (c) $2.0 \mathrm{~h}$.

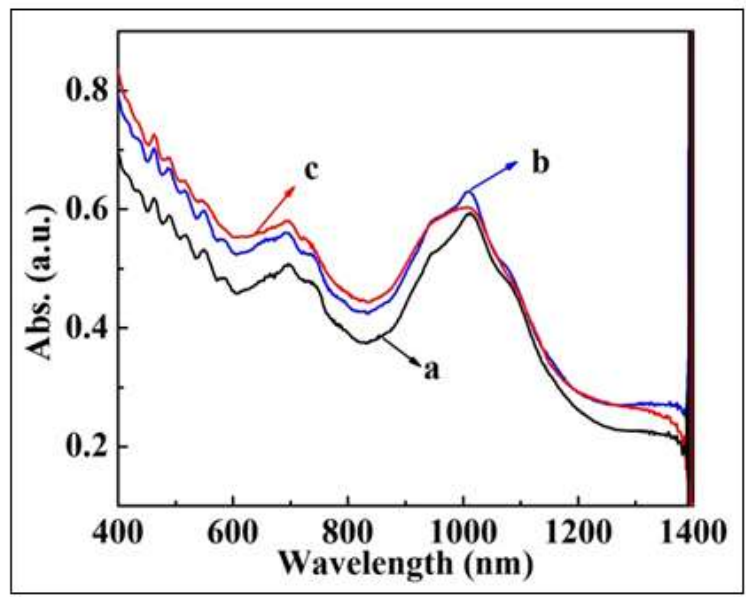

Fig-3: Vis-NIR spectra of dispersion solution of SWNTs with different centrifugal time (a) $1 \mathrm{~h}$, (b) $1.5 \mathrm{~h}$ and (c) $2.0 \mathrm{~h}$

As is shown in Figure-3, the absorbance of the solution of sodium deoxycholate-SWNTs varies with the centrifugal time. The absorption spectra line (b) presents better resolution and higher effective absorption intensity than those of the absorption spectra line (a) and (c), the relative absorption peak of line (b) is larger than those of line (a) and (c). So the centrifugal time $(1.5 \mathrm{~h})$ has a better dispersion effect on SWNTs. It means the dispersion of sodium deoxycholate towards SWNTs is related to the centrifugal time, when the centrifugal time is $1.5 \mathrm{~h}$, it has the best dispersion to SWNTs. When the the centrifugal time is $1.5 \mathrm{~h}$, the purification of SWCNTs was higher, the purification of SWCNTs was realized in the meanwhile. 


\section{Cyclic voltammetry curves of SWCNTs}

Comparing with SWCNTs bundles, dispersible

SWCNTs have an excellent electron transport capability, and they are widely used in sensor design [9]. In the experiment, the naked glassy carbon electrode was modified with SWCNTs before and after dispersion, and the influence of SWCNTs on the electron transfer ability was investigated by analyzing the cyclic voltammetry of the modified glassy carbon electrode in the three-electrode system.

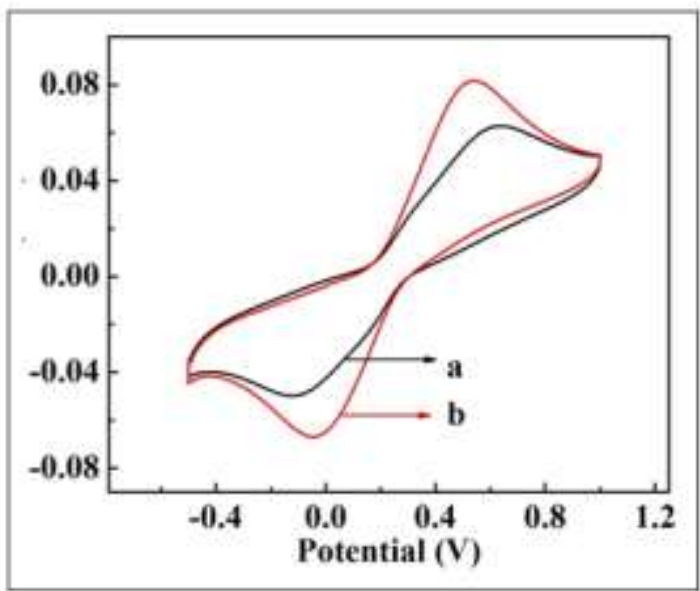

Fig-4: Cyclic voltammetry curve of (a) bare glass electrode and (b) sodium deoxycholate-SWNTs-bare glass electrode

As is shown in figure 4 , curve a is cyclic voltammetry curve of bare glass electrodes in buffer solution of PBS, curve b is cyclic voltammetry curve of glass electrodes modified by SWCNTs in buffer solution of PBS. It can be clearly observed that the peak of curve $b$ is significantly lower than that of curve a, and the peak area of curve $b$ is also significantly greater than that of curve a. This indicated that the electron transport speed of the working electrode was accelerated after the bare glass electrode was modified with porous SWCNTs. Compared with the pore surface area of bare glass electrodes is significantly larger, so the surface of the working electrode modified by SWCNTs presents a better porous structure, which improves the mass transfer effect of the modified working electrode. Thus, it was suggested that the conductivity of the dispersed SWCNTs was improved after sodium deoxycholate dispersion. It was further demonstrated that the dispersibility of SWCNTs in aqueous solution was significantly improved after the action of sodium deoxycholate in aqueous solution. Therefore, the preparation of water-dispersible SWCNTs by this method has potential research value in the field of electrochemistry.

\section{CONCLUSION}

SWCNTs were purified and dispersed in sodium deoxycholate solution by ultrasonic fragmentation, and the purified and dispersed SWCNTs in water were obtained by centrifugal separation. When SWCNTs were dispersed by sodium deoxycholate, the sodium deoxycholate formed non-covalent with SWCNTs, which weakened the van der Waals forces between SWCNTs bundles, the dispersibility of SWCNTs was achieved, and the dispersible solution of SWCNTs was obtained. Therefore, it provides a feasible method for obtaining highly purified SWCNTs that are dispersible in water. $\mathrm{CV}$ curve of the dispersion solution of SWCNTs was investigated by three electrode working system, which further demonstrated the good conductivity of the dispersion solution of SWCNTs. Therefore, water-dispersible and high-purity SWCNTs have potential research value in the field of electrochemistry.

\section{Acknowledgment}

This work was supported by the guiding science and technology plan project of Daqing (zd2019-44).

\section{REFERENCES}

1. Park, S., Pitner, G., Giri, G., Koo, J. H., Park, J., Kim, K., \& Bao, Z. (2015). Large- Area Assembly of Densely Aligned Single- Walled Carbon Nanotubes Using Solution Shearing and Their Application to Field- Effect Transistors. Advanced Materials. 27(16):2656-2662.

2. Syrgiannis, Z., Trautwein, G., Calvaresi, M., Modugno, G., Zerbetto, F., Carraro, M., \& Bonchio, M. (2019). Cover Feature: Controlling Size- Dispersion of Single Walled Carbon Nanotubes by Interaction with Polyoxometalates Armed with a Tryptophan Tweezer (Eur. J. Inorg. Chem. 3- 4/2019). European Journal of Inorganic Chemistry. 339-339.

3. Wang, R., Sun, J., Gao, L., \& Zhang, J. (2010). Dispersion of single-walled carbon nanotubes by DNA for preparing transparent conductive films. Journal of Materials Chemistry. 20(33):69036909.

4. Matsumoto, K., Takahashi, T., Jinguji, Y., \& Jikei, M. (2017). Dispersion of Single-Walled Carbon Nanotubes in Ketone Solvents and Effects of 
Sonication. International journal of the Society of Materials Engineering for Resources. 20-24.

5. Li, Y., Li, G., Wang, X., Zhu, Z., Ma, H., Zhang, T., \& Jin, J. (2012). Poly (ionic liquid)-wrapped single-walled carbon nanotubes for sub-ppb detection of CO2. Chemical Communications, 48(66), 8222-8224.

6. Pramanik, D., \& Maiti P. K. (2017). DNAAssisted Dispersion of Carbon Nanotubes and Comparison with Other Dispersing Agents. ACS Applied Materials \& Interfaces. 9(40): 3528735296.

7. Yu, H., Hermann, S., Schulz, S. E., Gessner, T.,
Dong, Z., \& Li, W. J. (2012). Optimizing sonication parameters for dispersion of singlewalled carbon nanotubes. Chemical Physics. 1116.

8. Min, L. (2013), Study on electrochemical properties of single conductive single walled carbon nanotubes. Harbin: heilongjiang university.

9. Cao, Q., \& Rogers, J. A. (2009). Ultrathin Films of Single- Walled Carbon Nanotubes for Electronics and Sensors: A Review of Fundamental and Applied Aspects. Advanced Materials. 21(1), 2953. 